Annals of Pure and Applied Mathematics

Vol. 13, No. 2, 2017, 273-292

ISSN: 2279-087X (P), 2279-0888(online)

Published on 16 May 2017

Annals of

www.researchmathsci.org

DOI: http://dx.doi.org/10.22457/apam.v13n2a14

Pure and Applied

Mathematics

\title{
Soft Intersection Ideals of Semiring
}

\section{P. Murugadas and M.R.Thirumagal}

Department of Mathematics, Annamalai University, Annamalainagar-608002, India

Corresponding author. Email: bodi_muruga@yahoo.com

Received 11 April 2017; accepted 23 April 2017

Abstract. Molodtsov introduced the concept of soft sets, which can be seen as a new Mathematical tool for dealing with uncertainty. In this paper, we initiate the study of soft intersection ideals of semirings by using the soft set theory. The notions of soft intersection semirings, soft intersection left(right, two-sided) ideals of semiring and soft intersection quasi and bi-ideals of semirings are introduced and several related properties are investigated.

Keywords: Soft set, soft intersection semirings, soft intersection left (right, two-sided) ideals of semiring and soft intersection quasi and bi-ideals of semirings.

AMS Mathematics Subject Classification (2010): 06D72, 16Y60, 16D25

\section{Introduction}

The notion of a semiring was introduced by Vandiver in 1934. Needless to say, semirings found their full place in Mathematics long before years. The applications of semirings to areas such as optimization theory, graph theory, generalized fuzzy computation, automata theory, formal language theory, coding theory and analysis of computer programs have been extensively studied in the literature (cf. [7,8]). It is also well-known that ideals usually play a fundamental role in algebra, especially in the study of rings. Nevertheless, ideals in a semiring $S$ do not in general coincide with the usual ring ideals if $\mathrm{S}$ is a ring, and so many results in ring theory have no analogues in semirings using only ideals. Consequently, some more restricted concepts of ideals such as k-ideals [9] and h-ideals [10] have been introduced in the study of the semiring theory. Moreover, the fuzzy set theory initiated by Zadeh [16] has been successfully applied to generalize many basic concepts in algebra. Rosenfeld [14] proposed the concept of group in order to establish the algebraic structure of fuzzy sets. In fact, several researchers have investigated a fuzzy theory in semirings. They introduced the notions of fuzzy semirings, fuzzy (prime) ideals, fuzzy k-ideals, fuzzy h-ideals and L-fuzzy ideals in semirings, and obtained many related results. However, all of these theories have their own difficulties which are pointed out in [13] by Molodtsov who then proposed a completely new approach for modeling vagueness and uncertainty, that is free from the difficulties. This so-called soft set theory has potential applications in many different fields. Maji et al. [11] firstly worked on detailed theoretical study of soft sets. After that, the properties and applications on the soft set theory have been studied by many authors (e.g. [1, 2, 3, 4, 6, 12, 15, 17]). Feng et al. [5] dealt with the algebraic structure of semirings by applying soft set theory and 


\section{P. Murugadas and M.R.Thirumagal}

defined the notion of a soft semiring and focused on the algebraic properties of soft semirings. In this paper, we make a new approch to the classical semiring theory via soft sets, with the concept of soft intersection semiring and soft intersection ideals of semirings.

\section{Preliminaries}

Molodtsov [13] defined the notion of a soft set in the following way: Let $U$ be an initial universe set and $E$ be a set of parameters. The power set of $U$ is denoted by $P(U)$ and $A$ is a subset of $\mathrm{U}$. A pair $(F, A)$ is called a soft set over $\mathrm{U}$, where $F: A \rightarrow P(U)$. For $e \in A, F(e)$ may be considered as the set of $e$-approximate elements of the soft set $(F, A)$. Clearly, a soft set is not a set. For illustration, Molodtsov considered several examples in [13].

Example 2.1. Let $U=\left\{c_{1}, c_{2}, c_{3}, c_{4}, c_{5}, c_{6}, c_{7}\right\}$ be the set of seven cars and $\mathrm{E}=\{$ expensive, fuel efficiency, spacious, maintenance free, ecofriendly, high security measure $\}$ are set of parameters. Let $(F, P)$ be a soft set representing the "suitable cars" given by $(F, P)=\left\{\right.$ expensivecars $=\left\{c_{2}, c_{3}, c_{5}, c_{7}\right\}$, fuel efficiency $=\left\{c_{1}, c_{2}, c_{3}, c_{4}\right\}$, spacious $=\left\{c_{4}, c_{5}, c_{6}, c_{7}\right\}$, maintenance free $=\left\{c_{2}, c_{4}, c_{6}, c_{7}\right\}$ ecofriendly $=\left\{c_{1}, c_{2}, c_{3}\right.$, , high security measure $\left.=\left\{c_{3}, c_{4}, c_{6}, c_{7}\right\}\right\}$. Suppose that $\mathrm{Mr} \mathrm{X}$ wants to buy a car consisting the parameter fuel efficiency, spacious, eco friendly, high security measure which forms the subset $P=\{$ fuel efficiency, spacious, eco friendly, high security measure of the set $E$. The problem is to select the car which is suitable with the choice parameters set by $\mathrm{Mr} \mathrm{X}$.

Definition 2.2. Let $f_{A}, f_{B} \in S(U)$. Then, $f_{A}$ is called a soft subset of $f_{B}$ and denoted by $f_{A} \subseteq f_{B}$ if $f_{A}(x) \subseteq f_{B}(x)$ for all $x \in E$.

Definition 2.3. Let $f_{A}, f_{B} \in S(U)$. Then, union of $f_{A}$ and $f_{B}$ denoted by $f_{A} \cup f_{B}$, is defined as $f_{A} \cup f_{B}=f_{A \cup B}$, where $f_{A \cup B}(x)=f_{A}(x) \cup f_{B}(x)$ for all $x \in E$.

Definition 2.4. Let $f_{A}, f_{B} \in S(U)$. Then, intersection of $f_{A}$ and $f_{B}$ denoted by $f_{A} \cap f_{B}$, is defined as $f_{A} \cap f_{B}=f_{A \cap B}$, where $f_{A \cap B}(x)=f_{A}(x) \cap f_{B}(x)$ for all $x \in E$.

Definition 2.5. Let $f_{A}, f_{B} \in S(U)$. Then, $\wedge$-product of $f_{A}$ and $f_{B}$ denoted by $f_{A} \wedge f_{B}$, is defined as $f_{A} \wedge f_{B}=f_{A \wedge B}$, where $f_{A \wedge B}(x, y)=f_{A}(x) \cap f_{B}(y)$ for all $(x, y) \in E \times E$.

Definition 2.6. Let $f_{A}$ and $f_{B}$ be soft sets over the common universe $\mathrm{U}$ and $\Psi$ be a function from $A$ to $\mathrm{B}$. Then, soft image of $f_{A}$ under $\Psi$, denoted by $\Psi\left(f_{A}\right)$, is a soft set over $U$ by 
Soft Intersection Ideals of Semiring

$\left(\Psi\left(f_{A}\right)\right)(b)=\left\{\begin{array}{c}\bigcup\left\{f_{A}(a) \mid a \in A \text { and } \Psi(a)=b\right\}, \text { if } \Psi^{-1}(b) \neq \varnothing, \\ 0, \text { otherwise }\end{array}\right.$

for all $b \in B$. And soft pre-image (or soft inverse image) of $f_{B}$ under $\Psi$, denoted by $\Psi^{-1}\left(f_{B}\right)$, is a soft set over $U$ by $\Psi^{-1}\left(f_{B}\right)(a)=f_{B}(\Psi(a))$ for all $a \in A$.

Definition 2.7.Let $f_{A}$ be a soft set over $U$ and $\alpha \subseteq U$. Then, upper $\alpha$-inclusion of $f_{A}$, denoted by $U\left(f_{A} ; \alpha\right)$, is defined as $U\left(f_{A} ; \alpha\right)=\left\{x \in A \mid f_{A}(x) \supseteq \alpha\right\}$.

\section{Soft Intersection sum, product and soft characteristic function}

In this section, we define soft intersection sum, product and soft characteristic function and study their properties.

Definition 3.1. Let $f_{S}$ and $g_{S}$ be soft sets over the common universe $U$. Then, soft intersection sum $f_{S}+g_{S}$ is defined by

$$
\left(f_{S}+g_{S}\right)(x)=\left\{\begin{array}{l}
\bigcup_{x=y+z}\left\{f_{S}(y) \cap g_{S}(z)\right\}, \text { if there exists } y, z \in S \\
\varnothing, \text { otherwise. }
\end{array}\right.
$$

for all $x \in S$.

Definition 3.2. Let $f_{S}$ and $g_{S}$ be soft sets over the common universe $U$. Then, soft intersection product $f_{S} \circ g_{S}$ is defined by

$$
\left(f_{S} \circ g_{S}\right)(x)=\left\{\begin{array}{l}
\bigcup_{x=y z}\left\{f_{S}(y) \cap g_{S}(z)\right\}, \text { if there exists } \mathrm{y}, \mathrm{z} \in \mathrm{S} \\
\varnothing, \text { otherwise. }
\end{array}\right.
$$

for all $x \in S$.

Example 3.3. Consider the semiring $S=\{0, a, b, c\}$ defined by the following table:

\begin{tabular}{|l|l|l|l|l|}
\hline$\oplus$ & 0 & $\mathrm{~A}$ & $\mathrm{~b}$ & $\mathrm{c}$ \\
\hline 0 & 0 & $\mathrm{~A}$ & $\mathrm{~b}$ & $\mathrm{c}$ \\
\hline $\mathrm{a}$ & $\mathrm{a}$ & $\mathrm{B}$ & $\mathrm{c}$ & $\mathrm{a}$ \\
\hline $\mathrm{b}$ & $\mathrm{b}$ & $\mathrm{C}$ & $\mathrm{a}$ & $\mathrm{b}$ \\
\hline $\mathrm{c}$ & $\mathrm{c}$ & $\mathrm{A}$ & $\mathrm{b}$ & $\mathrm{c}$ \\
\hline
\end{tabular}

\begin{tabular}{|l|l|l|l|l|}
\hline$\odot$ & 0 & $\mathrm{a}$ & $\mathrm{b}$ & $\mathrm{c}$ \\
\hline 0 & 0 & 0 & 0 & 0 \\
\hline $\mathrm{a}$ & 0 & $\mathrm{a}$ & $\mathrm{b}$ & $\mathrm{c}$ \\
\hline $\mathrm{b}$ & 0 & $\mathrm{~b}$ & $\mathrm{~b}$ & $\mathrm{c}$ \\
\hline $\mathrm{c}$ & 0 & $\mathrm{c}$ & $\mathrm{c}$ & $\mathrm{c}$ \\
\hline
\end{tabular}

Let $U=D_{3}=\left\{\langle x, y\rangle: x^{3}=y^{2}=e, x y=y x^{2}\right\}=\left\{e, x, x^{2}, y, y x, y x^{2}\right\}$ be the universal set. Let $f_{S}$ and $g_{S}$ be soft sets over $U$ such that $f_{S}(0)=\{e, x, y, y x\}, f_{S}(a)=$ $\left\{e, x, y^{2}\right\}, f_{S}(b)=\left\{e, y, y x^{2}\right\}, f_{S}(c)=\left\{e, x, x^{2}, y\right\}$ and $g_{S}(0)=\left\{e, y, y^{2}\right\}, g_{S}(a)=$ $\{e, x, y x\}, g_{S}(b)=\left\{e, y x, y x^{2}\right\}, g_{S}(c)=\{e, y, y x\} .\left(f_{S} \circ g_{S}\right)(a)=\left(f_{S}(a) \cap g_{S}(a)\right)$ $=\{e, x\}$, 
P. Murugadas and M.R.Thirumagal

$\left(f_{S} \circ g_{S}\right)(b)=\left(f_{S}(a) \cap g_{S}(b)\right) \cup\left(f_{S}(b) \cap g_{S}(a)\right) \cup\left(f_{S}(b) \cap g_{S}(b)\right)=\{e\} \cup\{e\} \cup\left\{e, y x^{2}\right\}=\left\{e, y x^{2}\right\}$,

$\left(f_{S} \circ g_{S}\right)(c)=\left(f_{S}(a) \cap g_{S}(c)\right) \cup\left(f_{S}(b) \cap g_{S}(c)\right) \cup\left(f_{S}(c) \cap g_{S}(a)\right) \cup\left(f_{S}(c) \cap g_{S}(b)\right) \cup\left(f_{S}(c) \cap g_{S}(c)\right)$

$=\{e\} \cup\{e, y\} \cup\{e, x\} \cup\{e\} \cup\{e\} \cup\{e, y\}$

$=\{e, x, y\}$.

Theorem 3.4. Let $f_{S}, g_{S}, h_{S} \in S(U)$. Then,

(1) $\left(f_{S} \circ g_{S}\right) \circ h_{S}=f_{S} \circ\left(g_{S} \circ h_{S}\right)$.

(2) $f_{S} \circ g_{S} \neq f_{S} \circ g_{S}$.

(3) $f_{S} \circ\left(g_{S} \cup h_{S}\right)=\left(f_{S} \circ g_{S}\right) \cup\left(f_{S} \circ h_{S}\right)$ and $\left(f_{S} \cup g_{S}\right) \circ h_{S}=\left(f_{S} \circ h_{S}\right) \cup\left(g_{S} \circ h_{S}\right)$.

(4) $f_{S} \circ\left(g_{S} \tilde{\cap} h_{S}\right)=\left(f_{S} \circ g_{S}\right) \tilde{\cap}\left(f_{S} \circ h_{S}\right)$ and $\left(f_{S} \tilde{\cap} g_{S}\right) \circ h_{S}=\left(f_{S} \circ h_{S}\right) \circ\left(g_{S} \circ h_{S}\right)$.

(5) If $f_{S} \tilde{\subseteq} g_{S}$, then $f_{S} \circ h_{S} \tilde{\subseteq} g_{S} \circ h_{S}$ and $h_{S} \circ f_{S} \tilde{\subseteq} h_{S} \circ g_{S}$.

(6) If $t_{S}, l_{S} \in S(U)$ such that $t_{S} \tilde{\subseteq} f_{S}$ and $l_{S} \tilde{\subseteq} g_{S}$, then $l_{S} \circ g_{S} \tilde{\subseteq} f_{S} \circ g_{S}$.

(7) $\left(f_{S}+g_{S}\right)+h_{S}=f_{S}+\left(g_{S}+h_{S}\right)$.

(8) $f_{S} \circ\left(g_{S}+h_{S}\right)=\left(f_{S} \circ g_{S}\right)+\left(f_{S} \circ h_{S}\right)$.

Proof: (1) and (2) follows from Definition 3.1 and Example 3.1.

(3) Let $a \in S$. If $a$ is not expressible as $a=x y$, then $f_{S} \circ\left(g_{S} \tilde{\cap} h_{S}\right)(A)=\varnothing$.

Similarly, $\left(\left(f_{S} \circ g_{S}\right) \tilde{\cap}\left(f_{S} \cup h_{S}\right)\right)(a)=\left(f_{S} \circ g_{S}\right)(a) \cup\left(f_{S} \cup h_{S}\right)(a)=\varnothing \cup \varnothing=\varnothing$.

Now, let there exist $x, y \in S$ such that $a=x y$.

$$
\begin{aligned}
& \left(f_{S} \circ\left(g_{S} \tilde{\cap} h_{S}\right)\right)(a)=\bigcup_{a=x y}\left(f_{S}(x) \cap\left(g_{S} \tilde{\cup} h_{S}\right)(y)\right) \\
& =\bigcup_{a=x y}\left[\left(f_{S}(x) \cap g_{S}(y)\right) \cup\left(f_{S}(x) \cap h_{S}(y)\right)\right] \\
& =\bigcup_{a=x y}\left[\left(f_{S}(x) \cap g_{S}(y)\right)\right] \cup\left[\bigcup_{a=x y}\left[\left(f_{S}(x) \cap h_{S}(y)\right)\right]\right. \\
& =\left(f_{S} \circ g_{S}\right)(a) \cup\left(f_{S} \circ h_{S}\right)(a) \\
& =\left[\left(f_{S} \circ g_{S}\right) \tilde{\cup}\left(f_{S} \circ h_{S}\right)\right](a) .
\end{aligned}
$$

Thus $\left(f_{S} \tilde{\cup} g_{S}\right) \circ h_{S}=\left(f_{S} \circ h_{S}\right) \tilde{\cup}\left(g_{S} \circ h_{S}\right)$.

Similarly, we can prove(4) is also clear.

(5) Let $x \in S$. If $x$ is not expressible as $x=y z$, then $\left(f_{S} \circ h_{S}\right)(x)=\left(g_{S} \circ h_{S}\right)(x)=\varnothing$. Otherwise, 


\section{Soft Intersection Ideals of Semiring}

$\left(f_{S} \circ h_{S}\right)(x)=\bigcup_{x=y z}\left(f_{S}(y) \cap h_{S}(z)\right)$ $\subseteq \bigcup_{x=y z}\left(g_{S}(y) \cap h_{S}(z)\right)$ (since $\left.f_{S}(y) \subseteq g_{S}(y)\right)=\left(g_{S} \circ h_{S}\right)(x)$.

Similarly, one can show that $h_{S} \circ f_{S} \subseteq h_{S} \circ g_{S}$.

(6) can be proved similar to (5).

(7) Let $x \in S$. If $x$ is not expressible as $x=y+z$, then

$\left(\left(f_{S}+g_{S}\right)+h_{S}\right)(x)=\varnothing$. Otherwise,

If $x=y+z$, then

$\left(\left(f_{S}+g_{S}\right)+h_{S}\right)(x)=\bigcup_{x=y+z}\left\{\left(f_{S}+g_{S}\right)(y) \cap h_{S}(z)\right\}$

$=\left\{\begin{array}{l}\bigcup_{x=y+z}\left\{\bigcup_{y=y_{1}+y_{2}}\left(f_{S}\left(y_{1}\right) \cap g_{S}\left(y_{2}\right)\right) \cap h_{S}(z)\right\} \\ \bigcup_{x=y+z}\left\{\varnothing \cap h_{S}(z)\right\}=\varnothing \text { if } y \neq y_{1}+y_{2}\end{array}=\left\{\begin{array}{l}\bigcup_{x=y+z} \bigcup_{y=y_{1}+y_{2}}\left(f_{S}\left(y_{1}\right) \cap g_{S}\left(y_{2}\right) \cap h_{S}(z)\right. \\ \varnothing, \text { otherwise }\end{array}\right.\right.$

$=\left\{\begin{array}{l}\bigcup_{x=y_{1}+y_{2}+z}\left(f_{S}\left(y_{1}\right) \cap g_{S}\left(y_{2}\right) \cap h_{S}(z)\right) \\ \varnothing, \text { otherwise }\end{array}=\left\{\begin{array}{l}\bigcup_{x=y_{1}+\left(y_{2}+z\right)}\left(f_{S}\left(y_{1}\right) \cap\left(g_{S}\left(y_{2}\right) \cap h_{S}(z)\right)\right. \\ \varnothing, \text { otherwise }\end{array}\right.\right.$

$\left(f_{S}+g_{S}\right)+h_{S}=f_{S}+\left(g_{S}+h_{S}\right)$.

(7) Let $x \in S$. If $x$ is not expressible as $x=y z$, then

$\left(f_{S} \circ\left(g_{S}+h_{S}\right)\right)(x)=\varnothing$. Otherwise,

If $x=y z$, then

$\left(f_{S} \circ\left(g_{S}+h_{S}\right)\right)(x)=\bigcup_{x=y z}\left\{\left(f_{S}(y) \cap\left(g_{S}+h_{S}\right)(z)\right)\right\}$

If $z \neq z_{1}+z_{2}$, then $\left(g_{S}+h_{S}\right)(z)=\varnothing$ and so

$\left(f_{S} \circ\left(g_{S}+h_{S}\right)\right)(x)=\varnothing$. Therefore assume $z=z_{1}+z_{2}$,

$=\left\{\begin{array}{l}\bigcup_{x=y\left(z_{1}+z_{2}\right)}\left\{f_{S}(y) \cap\left\{\bigcup_{z=z_{1}+z_{2}}\left(g_{S}\left(z_{1}\right) \cap h_{S}\left(z_{2}\right)\right)\right\}\right\} \\ \varnothing, \text { otherwise }\end{array}\right.$

$=\left\{\begin{array}{l}\bigcup_{x=y\left(z_{1}+z_{2}\right)}\left\{\left\{f_{S}(y) \cap g_{S}\left(z_{1}\right)\right\} \cup\left\{f_{S}(y) \cap\left(g_{S}\left(z_{2}\right)\right\}\right\}\right. \\ \varnothing, \text { otherwise }\end{array}\right.$ 
P. Murugadas and M.R.Thirumagal

$=\left\{\begin{array}{l}\bigcup_{\left.x=y z_{1}+y z_{2}\right)}\left\{\left\{\left(f_{S} \circ g_{S}\right) \cap\left(f_{S} \circ h_{S}\right)\left(y z_{2}\right)\right\}\right\} \\ \varnothing, \text { otherwise }\end{array}\right.$
$=\left(\left(f_{S} \circ g_{S}\right)+\left(f_{S} \circ h_{S}\right)\right)(x)$
$\Rightarrow f_{S} \circ\left(g_{S}+h_{S}\right)=\left(f_{S} \circ g_{S}\right)+\left(f_{S} \circ h_{S}\right)$.

Definition 3.5. Let $X$ be a subset of S. We denote by $S_{X}$ the soft characteristic function of $X$ and is define as

$S_{X}(x)= \begin{cases}U, & \text { if } x \in X, \\ \varnothing, & \text { if } x \notin X .\end{cases}$

It is obvious that the soft characteristic function is a soft set over $U$, that is, $S_{X}: S \rightarrow P(U)$.

Theorem 3.6. Let $X$ and $Y$ be nonempty subsets of a semiring $S$. Then, the following properties hold:

(i) if $X \subseteq Y$, then $S_{X} \subseteq S_{Y}$.

(ii) $S_{X} \tilde{\cap} S_{Y}=S_{X \cap Y}, S_{X} \tilde{\cup} S_{Y}=S_{X \cup Y}$.

(iii) $S_{X} \circ S_{Y}=S_{X Y}$.

(iv) $S_{X}+S_{Y}=S_{X+Y}$.

Proof: (i) is straight forward by Definition 3.3.

(ii) Let $s$ be any element of $S$. Suppose $s \in X \cap Y$. Then, $s \in X$ and $s \in Y$. Thus, we have

$\left(S_{X} \cap S_{Y}\right)(s)=S_{X}(s) \cap S_{Y}(s)=U \cap U=U=S_{X \cap Y}(s)$.

Suppose $s \notin X \cap Y$. Then, $s \notin X$ or $s \notin Y$. Hence, we have

$\left(S_{X} \tilde{\cap} S_{Y}\right)(s)=S_{X}(s) \cap S_{Y}(s)=\varnothing=S_{X \cap Y}(s)$.

Let $s$ be any element of $\mathrm{S}$. Suppose $s \in X \cup Y$. Then, $s \in X$ or $s \in Y$. Thus, we have $\left(S_{X} \cup S_{Y}\right)(s)=S_{X}(s) \cup S_{Y}(s)=U=S_{X \cup Y}(s)$.

Suppose $s \notin X \cup Y$. Then, $s \notin X$ and $s \notin Y$.

Hence, we have

$\left(S_{X} \cup S_{Y}\right)(s)=S_{X}(s) \cup S_{Y}(s)=\varnothing=S_{X \cup Y}(s)$.

(iii) Let $s$ be any element of $\mathrm{S}$. Suppose $s \in X Y$. Then, $s=x y$ for some $x \in X$ and $y \in Y$. Thus we have

$\left(S_{X} \circ S_{Y}\right)(s)=\bigcup_{s=x y}\left(S_{X}(x) \cap S_{Y}(y)\right) \supseteq S_{X}(x) \cap S_{Y}(y)=U$.

This implies that $\left(S_{X} \circ S_{Y}\right)(s)=U$. Since $s=x y \in X Y, S_{X Y}(s)=U$. Thus, 


\section{Soft Intersection Ideals of Semiring}

$S_{X} \circ S_{Y}=S_{X Y}$.

In another case, when $s \notin X Y$, we have $s \neq x y$ for all $x \in X$ and $y \in Y$. If $s=m n$ for some $m, n \in S$, then we have

$\left(S_{X} \circ S_{Y}\right)(s)=\bigcup_{s=m n}\left(S_{X}(m) \cap S_{Y}(n)\right)=\varnothing=S_{X Y}(s)$.

If $s \neq m n$ for all $m, n \in S$, then $\left(S_{X} \circ S_{Y}\right)(s)=\varnothing=S_{X Y}(s)$. Therefore in all the cases, we have $S_{X} \circ S_{Y}=S_{X Y}$.

\section{Soft intersection semiring}

Definition 4.1. Let $S$ be a semiring and $f_{S}$ be a soft set over $\mathrm{U}$. Then, $f_{S}$ is called a soft intersection semiring of S, if

(1) $f_{S}(x+y) \supseteq f_{S}(x) \cap f_{S}(y)$

(2) $f_{S}(x y) \supseteq f_{S}(x) \cap f_{S}(y)$ for all $x, y \in S$.

Example 4.2. Consider the semiring $\mathrm{S}=\{0, \mathrm{a}, \mathrm{b}, \mathrm{c}\}$ defined by the following table:

\begin{tabular}{|c|l|l|l|l|}
\hline$\oplus$ & 0 & $\mathrm{a}$ & $\mathrm{b}$ & $\mathrm{c}$ \\
\hline 0 & 0 & $\mathrm{a}$ & $\mathrm{b}$ & $\mathrm{c}$ \\
\hline $\mathrm{a}$ & $\mathrm{a}$ & $\mathrm{b}$ & $\mathrm{c}$ & $\mathrm{a}$ \\
\hline $\mathrm{b}$ & $\mathrm{b}$ & $\mathrm{c}$ & $\mathrm{a}$ & $\mathrm{b}$ \\
\hline $\mathrm{c}$ & $\mathrm{c}$ & $\mathrm{a}$ & $\mathrm{b}$ & $\mathrm{c}$ \\
\hline
\end{tabular}

\begin{tabular}{|l|l|l|l|l|}
\hline$\odot$ & 0 & $\mathrm{a}$ & $\mathrm{b}$ & $\mathrm{c}$ \\
\hline 0 & 0 & 0 & 0 & 0 \\
\hline $\mathrm{a}$ & 0 & $\mathrm{a}$ & $\mathrm{b}$ & $\mathrm{c}$ \\
\hline $\mathrm{b}$ & 0 & $\mathrm{~b}$ & $\mathrm{~b}$ & $\mathrm{c}$ \\
\hline $\mathrm{c}$ & 0 & $\mathrm{c}$ & $\mathrm{c}$ & $\mathrm{c}$ \\
\hline
\end{tabular}

Let $U=D_{3}=\left\{\langle x, y\rangle: x^{3}=y^{2}=e, x y=y x^{2}\right\}=\left\{e, x, x^{2}, y, y x, y x^{2}\right\}$ be the universal set. Let $f_{S}$ and $g_{S}$ be soft sets over $U$ such that $f_{S}(0)=\{e, x, y, y x\}$, $f_{S}(a)=\left\{e, x, y^{2}\right\}, f_{S}(b)=\left\{e, y, y x^{2}\right\}, f_{S}(c)=\left\{e, x, x^{2}, y\right\}$

Clearly $f_{-} S$ is a SI-semiring over $\mathrm{U}$.

It is easy that if $f_{-} S=U$ for all $x \in S$, then $f_{S}$ is a SI-semiring over $\mathrm{U}$. We denote that such a kind of SI-semiring by $\tilde{S}$. It is obvious that $\widetilde{S}=S_{s}$, that is $S_{x}(x)=U$ for all $x \in S$.

Lemma 4.3. Let $f_{S}$ be any SI-semiring over U. Then, we have the following:

(2) $f_{S} \circ \tilde{S} \widetilde{\subseteq} \tilde{S}$ and $\widetilde{S} \circ f_{S} \widetilde{\subseteq} \widetilde{S}$.

(1) $\widetilde{S} \circ \tilde{S} \widetilde{\subseteq}$.

(3) $f_{S} \widetilde{\cup} \tilde{S} \widetilde{=S}$ and $\widetilde{S} \widetilde{\cap} f_{S} \widetilde{\subseteq} \widetilde{S}$.

(4) $\widetilde{S}+\tilde{S} \widetilde{\subseteq}$.

(5) $f_{S}+\tilde{S} \widetilde{\subseteq}$ and $\widetilde{S}+f_{S} \widetilde{\subseteq}$.

Proof: Obviously (1),(2) and (3) are true.

(4) for any $x \in S$

$(\tilde{S}+\tilde{S})(\mathrm{x})=\mathrm{U}_{\mathrm{x}=\mathrm{a}+\mathrm{b}}(\tilde{S}(a) \cap \widetilde{S}(\mathrm{~b}))$

$=\mathrm{U}_{\mathrm{x}=\mathrm{a}+\mathrm{b}}(\mathrm{U}) \subseteq U=\tilde{S}(\mathrm{x})$ 
P. Murugadas and M.R.Thirumagal

$(5)\left(f_{S}+\tilde{S}\right)(x)=\bigcup_{x=a+b}\left(f_{S}(a) \cap \tilde{S}(b)\right)=\bigcup_{x=a+b}\left(f_{S}(a)\right) \subseteq \bigcup_{x=a+b}(U)=\bigcup_{x=a+b} \tilde{S}(x)$ $\left(f_{S}+\tilde{S}\right)(x) \subseteq \tilde{S}(x) \Rightarrow\left(f_{S}+\tilde{S}\right) \subseteq \tilde{S}$.

Similarly, we can prove $\tilde{S}+f_{S} \subseteq \tilde{S}$.

Theorem 4.4. Let $f_{S}$ be a soft set over $U$. Then, $f_{S}$ is a SI-semiring over $U$ if and only if

(1) $f_{S}+f_{S} \tilde{\subset} f_{S}$.

(2) $f_{S} \circ f_{S} \tilde{\subseteq} f_{S}$.

Proof: Assume that $f_{S}$ is a SI-semiringover $U$. Let $a \in S$. If $\left(f_{S}+f_{S}\right)(a)=\varnothing$, then it is obvious that $\left(f_{S}+f_{S}\right)(a) \subseteq f_{S}(a)$, thus $f_{S}+f_{S} \subseteq f_{S}$.

Otherwise, there exist elements $x, y \in S$ such that $a=x+y$. Then, since $f_{S}$ is a SIsemiring over $\mathrm{U}$, we have:

$$
\begin{aligned}
& \text { (1) } \quad\left(f_{S}+f_{S}\right)(a)=\bigcup_{a=x+y}\left(f_{S}(x) \cap f_{S}(y)\right) \\
& \subseteq \bigcup_{a=x+y} f_{S}(x+y)=\bigcup_{a=x+y} f_{S}(a)=f_{S}(a) .
\end{aligned}
$$

Thus, $f_{S}+f_{S} \tilde{\subseteq} f_{S}$.

(2) is similar to (1).

Conversely, assume that (1) and (2) are true. Let $x, y \in S$ and $a=x+y$. Then, we have

$$
\begin{aligned}
& f_{S}(x+y)=f_{S}(a) \supseteq\left(f_{S}+f_{S}\right)(a)=\bigcup_{a=x+y}\left(f_{S}(x) \cap f_{S}(y) \supseteq f_{S}(x) \cap f_{S}(y) .\right. \\
& f_{S}(x+y) \supseteq f_{S}(x) \cap f_{S}(y) .
\end{aligned}
$$

Similarly, $f_{S}(x y) \supseteq f_{S}(x) \cap f_{S}(y)$

Hence, $f_{S}$ is an SI-semiring over $\mathrm{U}$.

Theorem 4.5. Let $X$ be a nonempty subset of a semiring $S$. Then, $X$ is a subsemiring of $S$ if and only if $S_{X}$ is a SI-semiring of S.

Proof: Assume that $\mathrm{X}$ is a subsemiring of $\mathrm{S}$, that is, $X X \subseteq X$ and $X+X \subseteq X$. Then, we have

$S_{X}+S_{X}=S_{X+X} \subseteq S_{X}$ and $S_{X} S_{X} \Rightarrow S_{X X} \subseteq S_{X}$ (by Theorem 3.2(iii) and Theorem 3.2(iv)) and so $S_{X}$ is a SI-semiring over $\mathrm{U}$.

Conversely, let $x \in X+X$ and $S_{X}$ be a SI-semiring of $S$. Then, by Theorem 4.1, $S_{X}(x) \supseteq\left(S_{X}+S_{X}\right)(x)=\bigcup_{x=a+b}\left(S_{X}(a) \cap S_{X}(b)\right) \Rightarrow S_{X+X}(x)=U$ implying that $S_{X}(x)$ 
Soft Intersection Ideals of Semiring

$=U$, hence $X+X \subseteq X$ and let $x \in X$ and $S_{X}$ be a SI-semiring of $S$. Then, $S_{X}(x) \supseteq\left(S_{X} \circ S_{X}\right)(x)=S_{X X}(x)=U$ implying that $S_{X}(x)=U$. Hence $X X \subseteq X$ and so, $X$ is a subsemiring of $\mathrm{S}$.

Proposition 4.6. Let $f_{S}$ and $f_{T}$ be a SI-semiring over $\mathrm{U}$. Then, $f_{S} \wedge f_{T}$ is a SI-semiring over U.

Proof: Let $\left(x_{1}, y_{1}\right),\left(x_{2}, y_{2}\right) \in S \times T$. Then,

$$
\begin{aligned}
& f_{S \wedge T}\left(\left(x_{1}, y_{1}\right)+\left(x_{2}, y_{2}\right)\right)=f_{S \wedge T}\left(x_{1}+x_{2}, y_{1}+y_{2}\right)=f_{S}\left(x_{1}+x_{2}\right) \cap f_{T}\left(y_{1}+y_{2}\right) \\
& \supseteq\left[f_{S}\left(x_{1}\right) \cap f_{S}\left(x_{2}\right)\right] \cap\left[f_{T}\left(y_{1}\right) \cap f_{T}\left(y_{2}\right)\right]=\left[f_{S}\left(x_{1}\right) \cap f_{T}\left(y_{1}\right)\right] \cap\left[f_{S}\left(x_{2}\right) \cap f_{T}\left(y_{2}\right)\right] \\
& =f_{S \wedge T}\left(x_{1}, y_{1}\right) \cap f_{S \wedge T}\left(\left(x_{2}, y_{2}\right)\right. \\
& \text { and } \\
& f_{S \wedge T}\left(\left(x_{1}, y_{1}\right)\left(x_{2}, y_{2}\right)\right)=f_{S \wedge T}\left(x_{1} x_{2}, y_{1} y_{2}\right)=f_{S}\left(x_{1} x_{2}\right) \cap f_{T}\left(y_{1} y_{2}\right) \\
& \supseteq\left[f_{S}\left(x_{1}\right) \cap f_{S}\left(x_{2}\right)\right] \cap\left[f_{T}\left(y_{1}\right) \cap f_{T}\left(y_{2}\right)\right]=\left[f_{S}\left(x_{1}\right) \cap f_{T}\left(y_{1}\right)\right] \cap\left[f_{S}\left(x_{2}\right) \cap f_{T}\left(y_{2}\right)\right] \\
& =f_{S \wedge T}\left(x_{1}, y_{1}\right) \cap f_{S \wedge T}\left(\left(x_{2}, y_{2}\right) .\right.
\end{aligned}
$$

Therefore, $f_{S} \wedge f_{T}$ is a SI-semiring over $\mathrm{U}$.

Definition 4.7. Let $f_{S}, f_{T}$ be a SI-semirings over $U$. Then, the product of soft intersection semirings $f_{S}$ and $f_{T}$ isdefined as $f_{S} \times f_{T}=f_{S \times T}$ where $f_{S \times T}(x, y)=f_{S}(x) \times f_{T}(y)$ for all $(x, y) \in S \times T$.

Proposition 4.8. If $f_{S}$ and $f_{T}$ are SI-semiring over $\mathrm{U}$. Then, so is $f_{S} \times f_{T}$ over $U \times U$.

Proof: By Definition 4.2, $f_{S} \times f_{T}=f_{S \times T}$ where $f_{S \times T}(x, y)=f_{S}(x) \times f_{T}(y)$ for all $(x, y) \in S \times T$.

Then, for all $\left(x_{1}, y_{1}\right),\left(x_{2}, y_{2}\right) \in S \times T, f_{S \times T}\left(\left(x_{1}, y_{1}\right)+\left(x_{2}, y_{2}\right)\right)=f_{S \times T}\left(x_{1}+x_{2}, y_{1}+y_{2}\right)$

$=f_{S}\left(x_{1}+x_{2}\right) \times f_{T}\left(y_{1}+y_{2}\right) \supseteq\left[f_{S}\left(x_{1}\right) \cap f_{S}\left(x_{2}\right)\right] \times\left[f_{T}\left(y_{1}\right) \cap f_{T}\left(y_{2}\right)\right]$

$=\left[f_{S}\left(x_{1}\right) \times f_{T}\left(y_{1}\right)\right] \cap\left[f_{S}\left(x_{2}\right) \times f_{T}\left(y_{2}\right)\right]=f_{S \times T}\left(x_{1}, y_{1}\right) \cap f_{S \times T}\left(\left(x_{2}, y_{2}\right)\right.$

and

$f_{S \times T}\left(\left(x_{1}, y_{1}\right)\left(x_{2}, y_{2}\right)\right)=f_{S \times T}\left(x_{1} x_{2}, y_{1} y_{2}\right)=f_{S}\left(x_{1} x_{2}\right) \times f_{T}\left(y_{1} y_{2}\right)$

$\supseteq\left[f_{S}\left(x_{1}\right) \cap f_{S}\left(x_{2}\right)\right] \times\left[f_{T}\left(y_{1}\right) \cap f_{T}\left(y_{2}\right)\right]=\left[f_{S}\left(x_{1}\right) \times f_{T}\left(y_{1}\right)\right] \cap\left[f_{S}\left(x_{2}\right) \times f_{T}\left(y_{2}\right)\right]$

$=f_{S \times T}\left(x_{1}, y_{1}\right) \cap f_{S \times T}\left(\left(x_{2}, y_{2}\right)\right)$.

Therefore, $f_{S} \times f_{T}=f_{S \times T}$ is a SI-semiring over $U \times U$.

Proposition 4.9. If $f_{S}$ and $h_{S}$ are SI-semiring over U. Then, so is $f_{S} \tilde{\cap} h_{S}$ over U.

Proof: Let $x, y \in S$, then

$$
\left(f_{S} \tilde{\cap} h_{S}\right)(x+y)=f_{S}(x+y) \cap h_{S}(x+y) \supseteq\left(f_{S}(x) \cap f_{S}(y)\right) \cap\left(h_{S}(x) \cap h_{S}(y)\right)
$$


P. Murugadas and M.R.Thirumagal

$=\left(f_{S}(x) \cap h_{S}(x)\right) \cap\left(f_{S}(y) \cap h_{S}(y)\right)=\left(f_{s} \tilde{\cap} h_{S}\right)(x) \cap\left(f_{S} \tilde{\cap} h_{S}\right)(y)$

and

$\left(f_{S} \tilde{\cap} h_{S}\right)(x y)=f_{S}(x y) \cap h_{S}(x y) \supseteq\left(f_{S}(x) \cap f_{S}(y)\right) \cap\left(h_{S}(x) \cap h_{S}(y)\right)$

$=\left(f_{S}(x) \cap h_{S}(x)\right) \cap\left(f_{S}(y) \cap h_{S}(y)\right)=\left(f_{S} \tilde{\cap} h_{S}\right)(x) \cap\left(f_{S} \tilde{\cap} h_{S}\right)(y)$.

Therefore, $f_{S} \cap h_{s}$ is a SI-semiring over $\mathrm{U}$.

Proposition 4.10. Let $f_{S}$ be a soft set over $U$ and $\alpha$ be a subset of $U$ such that $\alpha \in \operatorname{Im}\left(f_{S}\right)$, where $\operatorname{Im}\left(f_{S}\right)=\left\{\alpha \subseteq U: f_{S}(x)=\alpha\right.$, for $\left.x \in S\right\}$. If $f_{S}$ is a SI-semiring over $U$, then $U\left(f_{S} ; \alpha\right)$ is a subsemiring of $S$.

Proof: Let $f_{S}(x)=\alpha$ for some $x \in S$, then $\varnothing \neq U\left(f_{S} ; \alpha\right) \subseteq S$. Let $x, y \in U\left(f_{S} ; \alpha\right)$, then $f_{S}(x) \supseteq \alpha$ and $f_{S}(y) \supseteq \alpha$. We need to show that $x y \in U\left(f_{S} ; \alpha\right)$ and $x+y \in U\left(f_{S}, \alpha\right)$. Since $f_{S}$ is a SI-semiring over $\mathrm{U}$, it follows that

$f_{S}(x y) \supseteq f_{S}(x) \cap f_{S}(y) \supseteq \alpha \cap \alpha=\alpha \Rightarrow x y \in U\left(f_{S} ; \alpha\right)$.

$f_{S}(x+y) \supseteq f_{S}(x) \cap f_{S}(y) \supseteq \alpha \cap \alpha=\alpha$.

This shows $x+y \in U\left(f_{S} ; \alpha\right)$.

Definition 4.11. Let $f_{S}$ be a SI-semiring over $\mathrm{U}$. Then, the subsemiring $U\left(f_{S} ; \alpha\right)$ are called upper $\alpha$-subsemiring of $f_{S}$.

Proposition 4.12. Let $f_{S}$ be a soft set over $\mathrm{U}, U\left(f_{S} ; \alpha\right)$ be upper $\alpha$-subsemiring of $f_{S}$ for each $\alpha \subseteq U$ and $\operatorname{Im}\left(f_{S}\right)$ be an ordered set by inclusion. Then, $f_{S}$ is a SI-semiring over U.

Proof: Let $x, y \in S$ and $f_{S}(x)=\alpha_{1}$ and $f_{S}(y)=\alpha_{2}$. Suppose that $\alpha_{1} \subseteq \alpha_{2}$. It is obvious that $x \in U\left(f_{S} ; \alpha_{1}\right)$ and $y \in U\left(f_{S} ; \alpha_{2}\right)$. Since $\alpha_{1} \subseteq \alpha_{2}, x, y \in U\left(f_{S} ; \alpha_{1}\right)$ and since $U\left(f_{S} ; \alpha\right)$ is a subsemiring of $S$ for all $\alpha \subseteq U$, it follows that $x y \in U\left(f_{S} ; \alpha_{1}\right), x+y \in U\left(f_{S}, \alpha_{1}\right)$. Hence, $f_{S}(x+y) \supseteq \alpha_{1}=\alpha_{1} \cap \alpha_{2}=f_{S}(x) \cap f_{S}(y)$ and $f_{S}(x y) \supseteq \alpha_{1}=\alpha_{1} \cap \alpha_{2}=f_{S}(x) \cap f_{S}(y)$. Thus, $f_{S}$ is a SI-semiring over $\mathrm{U}$.

Proposition 4.13. Let $f_{S}$ and $f_{T}$ be soft sets over $U$ and $\phi$ be a semiring isomorphism from $S$ to T. If $f_{S}$ is a SI-semiring over $\mathrm{U}$, then so is $\phi\left(f_{S}\right)$.

Proof: Let $t_{1}, t_{2} \in T$. Since $\phi$ is surjective, then there exists $s_{1}, s_{2} \in S$ such that $\phi\left(s_{1}\right)=t_{1}$ and $\phi\left(s_{2}\right)=t_{2}$. Then,

$\left(\phi\left(f_{S}\right)\right)\left(t_{1} t_{2}\right)=\bigcup\left\{f_{S}(s): s \in S, \phi(s)=t_{1} t_{2}\right\}$

$=\bigcup\left\{f_{S}(s): s \in S, s=\phi^{-1}\left(t_{1} t_{2}\right)\right\}=\bigcup\left\{f_{S}(s): s \in S, s=\phi^{-1}\left(\phi\left(s_{1} s_{2}\right)\right)=s_{1} s_{2}\right\}$ 
Soft Intersection Ideals of Semiring

$$
\begin{aligned}
& =\bigcup\left\{f_{S}\left(s_{1} s_{2}\right): s_{i} \in S, \phi\left(s_{i}\right)=t_{i}, i=1,2\right\} \\
& \supseteq \bigcup\left\{f_{S}\left(s_{1}\right) \cap f_{S}\left(s_{2}\right): s_{i} \in S, \phi\left(s_{i}\right)=t_{i}, i=1,2\right\} \\
& =\left(\bigcup\left\{f_{S}\left(s_{1}\right): s_{1} \in S, \phi\left(s_{1}\right)=t_{1}\right\}\right) \cap\left(\bigcup\left\{f_{S}\left(s_{2}\right): s_{2} \in S, \phi\left(s_{2}\right)=t_{2}\right\}\right) \\
& =\left(\phi\left(f_{S}\right)\right)\left(t_{1}\right) \cap\left(\phi\left(f_{S}\right)\right)\left(t_{2}\right) \text { and } \\
& \left(\phi\left(f_{S}\right)\right)\left(t_{1}+t_{2}\right)=\bigcup\left\{f_{S}(s): s \in S, \phi(s)=t_{1}+t_{2}\right\}=\bigcup\left\{f_{S}(s): s \in S, s=\phi^{-1}\left(t_{1}+t_{2}\right)\right\} \\
& =\bigcup\left\{f_{S}(s): s \in S, s=\phi^{-1}\left(\phi\left(s_{1}+s_{2}\right)\right)=s_{1}+s_{2}\right\} \\
& =\bigcup\left\{f_{S}\left(s_{1}+s_{2}\right) ; s_{i} \in S, \phi\left(s_{i}\right)=t_{i}, i=1,2\right\} \\
& \supseteq \bigcup\left\{f_{s}\left(s_{1}\right) \cap f_{S}\left(s_{2}\right) ; s_{i} \in S, \phi\left(s_{i}\right)=t_{i}, i=1,2\right\} \\
& =\left(\bigcup\left\{f_{S}\left(s_{1}\right): s_{1} \in S, \phi\left(s_{1}\right)=t_{1}\right\}\right) \cap\left(\bigcup\left\{\left(f_{S}\left(s_{2}\right), s_{2} \in S, \phi\left(s_{2}\right)=t_{2}\right)\right\}\right) \\
& =\phi\left(f_{S}\right)\left(t_{1}\right) \cap\left(\phi\left(f_{S}\right)\right)\left(t_{2}\right) .
\end{aligned}
$$

Hence $\phi\left(f_{S}\right)$ is a SI-semiring over $\mathbf{U}$.

Proposition 4.14. Let $f_{S}$ and $f_{T}$ be soft sets over $U$ and $\phi$ be a semiring homomorphism from $S$ to T. If $f_{T}$ is a SI-semiring over $\mathrm{U}$, then so is $\phi^{-1}\left(f_{T}\right)$.

Proof: Let $s_{1}, s_{2} \in S$. Then,

$$
\begin{aligned}
& \left.\phi^{-1}\left(f_{T}\right)\left(s_{1} s_{2}\right)=f_{T}\left(\phi\left(s_{1} s_{2}\right)\right)=f_{t}\left(\phi\left(s_{1}\right) \phi\left(s_{2}\right)\right)\right) \supseteq f_{T}\left(\phi\left(s_{1}\right)\right) \cap f_{T}\left(\phi\left(s_{2}\right)\right) \\
& \phi^{-1}\left(f_{T}\right)\left(s_{1} s_{2}\right)=\left(\phi^{-1}\left(f_{T}\right)\right)\left(s_{1}\right) \cap\left(\phi^{-1}\left(f_{T}\right)\right)\left(s_{2}\right)
\end{aligned}
$$

Let $s_{1}, s_{2} \in S$. Then,

$$
\begin{aligned}
& \phi^{-1}\left(f_{T}\right)\left(s_{1}+s_{2}\right)=f_{T}\left(\phi\left(s_{1}+s_{2}\right)\right)=f_{T}\left(\left(\phi\left(s_{1}\right)\right)+\left(\phi\left(s_{2}\right)\right)\right) \supseteq f_{T}\left(\phi\left(s_{1}\right)\right) \cap f_{T}\left(\phi\left(s_{2}\right)\right) \\
& \phi^{-1}\left(f_{T}\right)\left(s_{1}+s_{2}\right) \supseteq\left(\phi^{-1}\left(f_{T}\right)\left(s_{1}\right) \cap \phi^{-1}\left(f_{T}\right)\left(s_{2}\right)\right) .
\end{aligned}
$$

Hence, $\phi^{-1}\left(f_{T}\right)$ is an SI- semiring over $\mathrm{U}$.

\section{Soft intersection left (right, two-sided) ideals of semiring}

Definition 5.1. A soft set $f_{S}$ over $U$ is called a soft intersection left (right, two-sided) ideals of $S$ over $U$ if

(1) $f_{S}(x+y) \supseteq f_{S}(x) \cap f_{S}(y)$

(2) $f_{S}(x y) \supseteq f_{S}(x)\left(f_{s}(x y) \supseteq f_{S}(y)\right)$

for all $x, y \in S$. A soft set over $U$ is called a soft intersection two-sided ideal (soft intersection ideal) of $S$ if it is both soft intersection left and soft intersection right ideal of $S$ over $\mathrm{U}$.

Example 5.2. Consider the semiring $S=\{0, x, 1\}$ defined by the following table: 
P. Murugadas and M.R.Thirumagal

\begin{tabular}{|l|l|l|l|}
\hline$\oplus$ & 0 & $\mathrm{x}$ & 1 \\
\hline 0 & 0 & $\mathrm{x}$ & 1 \\
\hline $\mathrm{x}$ & $\mathrm{X}$ & 0 & $\mathrm{x}$ \\
\hline 1 & 1 & $\mathrm{x}$ & 0 \\
\hline
\end{tabular}

\begin{tabular}{|l|l|l|l|}
\hline$\odot$ & 0 & $\mathrm{x}$ & 1 \\
\hline 0 & 0 & 0 & 0 \\
\hline $\mathrm{x}$ & 0 & $\mathrm{x}$ & $\mathrm{x}$ \\
\hline 1 & 0 & $\mathrm{x}$ & 1 \\
\hline
\end{tabular}

Let $f_{S}$ be soft set over $S$ such that $f_{S}(0)=\{0, x, 1\}, f_{S}(x)=\{0, x\}, f_{S}(1)=\{x\}$. Then, one can easily show that $f_{S}$ is a SI-ideal of $S$ over U.

Theorem 5.3. Let $f_{S}$ be a SI-semiring over $U$. Then, $f_{S}$ is a SI-left ideal of $S$ over $U$ if and only if

(1) $f_{S}+f_{S} \subseteq f_{S}$.

(2) $S \circ f_{S} \subseteq f_{S}$.

Proof: Assume that $f_{S}$ is a SI-left ideal of $S$ over $U$. Let $a \in S$. If $\left(f_{S}+f_{S}\right)(a)=\varnothing$, then it is obvious that $\left(f_{S}+f_{S}\right)(a) \subseteq f_{S}(a)$, thus $f_{S}+f_{S} \subseteq f_{S}$.

Otherwise, there exist elements $x, y \in S$ such that $a=x+y$. Then, since $f_{S}$ is a SI-left ideal of $S$ over $\mathrm{U}$, we have:

$$
\left(f_{S}+f_{S}\right)(a)=\bigcup_{a=x+y}\left(f_{S}(x) \cap f_{S}(y)\right) \subseteq \bigcup_{a=x+y} f_{S}(x+y)=\bigcup_{a=x+y} f_{S}(a)=f_{S}(a)
$$

Thus, $f_{S}+f_{S} \tilde{\subseteq} f_{S}$.

(2) If $\left(\tilde{S} \circ f_{S}\right)(a)=\varnothing$, then it is obvious that

$\left(\tilde{S} \circ f_{S}\right)(a) \subseteq f_{S}(a)$, thus $\tilde{S} \circ f_{S} \subseteq f_{S}$.

Otherwise, there exist elements $x, y \in S$ such that $a=x y$. Then, since $f_{S}$ is a SI-left ideal of $S$ over $\mathrm{U}$, we have:

$$
\left(\tilde{S} \circ f_{S}\right)(a)=\bigcup_{a=x y}\left(\tilde{S}(x) \cap f_{S}(y)\right) \subseteq \bigcup_{a=x y}\left(U \cap f_{S}(x y)\right)=\bigcup_{a=x y}\left(U \cap f_{S}(a)\right)=f_{S}(a)
$$

Thus, $\tilde{S} \circ f_{S} \subseteq f_{S}$.

Conversely, assume that (1) and (2) are true. Let $x, y \in S$ and $a=x+y$. Then,we have:

$$
f_{S}(x+y)=f_{S}(a) \supseteq\left(f_{S}+f_{S}\right)(a)=\bigcup_{a=x+y}\left(f_{S}(x) \cap f_{S}(y) \supseteq f_{S}(x) \cap f_{S}(y) .\right.
$$

Similarly, $f_{S}(x y) \supseteq f_{S}(y)$

Hence, $f_{S}$ is an SI-left ideal over $\mathrm{U}$. This competes the proof.

Theorem 5.4. Let $f_{S}$ be a SI-semiring over $U$. Then, $f_{S}$ is a SI-right ideal of $S$ over $U$ if and only if

(1) $f_{S}+f_{S} \subseteq f_{S}$. 


\section{Soft Intersection Ideals of Semiring}

(2) $f_{S} \circ \tilde{S} \subseteq f_{S}$.

Proof: Similar to the proof of Theorem 5.1.

Corollary 5.5. $\tilde{S}$ is both SI-right and SI-left ideal of $S$.

Proof: Follows from Lemma 4.1-(1).

Theorem 5.6. Let $X$ be a nonempty subset of a semiring $S$. Then, $X$ is a left (right, two-sided) ideal of $S$ if and onlyif $S_{X}$ is an SI-left (right, two-sided) ideal of $S$ over $U$.

Proof: We give the proof for the SI-left ideals. Assume that $X$ is a left ideal of $S$, that is, $X+X \subseteq X \quad$ and $\quad S X \subseteq X$. Then, we have $S_{X}+S_{X}=S_{X+X} \subseteq S_{X}$ and $\tilde{S} \circ S_{X}=S_{S} \circ S_{X}=S_{S X} \subseteq S_{X}$. Thus, $S_{X}$ is an SI-left ideal of $S$ over $U$ by Theorem 5.1 .

Conversely, let $x \in S X$ and $S_{X}$ be an SI-left ideal of $S$ over U. Then, $S_{X}(x) \supseteq\left(\tilde{S} \circ S_{X}\right)(x)=\left(S_{S} \circ S_{X}\right)(x)=S_{S X}(x)=U$ implying that $S_{X}(x)=U$, hence $x \in X$. Similarly $S_{X}(x) \supseteq\left(S_{X}+S_{X}\right)(x+x)$. Thus, $S_{X} \subseteq X$ and $X$ is a left ideal of $\mathrm{S}$.

Theorem 5.7. Let $f_{S}$ and $g_{S}$ be SI-left (right) ideals of a semiring S. Then $f_{S}+g_{S}$ is a SI-left (right) ideal of S.

Proof: Suppose $f_{S}, g_{S}$ are SI-left ideals of a semiring $S$ and $x, y \in S$. If $\left(f_{S}+g_{S}\right)(x)=\varnothing$ or $\left(f_{S}+g_{S}\right)(y)=\varnothing$ then, $\left(f_{S}+g_{S}\right)(x) \cap\left(f_{S}+g_{S}\right)(y)=\varnothing \subseteq$ $\left(f_{S}+g_{S}\right)(x+y)$. If $\left(f_{S}+g_{S}\right)(x) \neq \varnothing$ and $\left(f_{S}+g_{S}\right)(y) \neq \varnothing$ then, $\left(f_{s}+g_{S}\right)(y)=$ $\bigcup_{y=c+d}\left\{f_{S}(c) \cap g_{S}(d)\right\}$.

Thus,

$$
\begin{aligned}
& \left(f_{S}+g_{S}\right)(x) \cap\left(f_{S}+g_{S}\right)(y)=\left(\bigcup_{x=a+b}\left\{f_{S}(a) \cap g_{S}(b)\right\}\right) \cap\left(\bigcup_{y=c+d}\left\{f_{S}(c) \cap g_{S}(d)\right\}\right) \\
& =\bigcup_{x=a+b} \bigcup_{y=c+d}\left\{\left(f_{S}(a) \cap g_{S}(b)\right) \cap\left(f_{S}(c) \cap g_{S}(d)\right)\right\} \\
& =\bigcup_{x=a+b} \bigcup_{y=c+d}\left\{\left(f_{S}(a) \cap f_{S}(c)\right) \cap\left(g_{S}(b) \cap g_{S}(d)\right)\right\} \\
& \subseteq \bigcup_{x=a+b} \bigcup_{y=c+d}\left\{\left(f_{S}(a+c) \cap g_{S}(b+d)\right)\right\} \\
& \subseteq\left(f_{S}+g_{S}\right)(x+y) .
\end{aligned}
$$

Again, if $\left(f_{S}+g_{S}\right)(x)=\varnothing$ then $\left(f_{S}+g_{S}\right)(x) \subseteq\left(f_{S}+g_{S}\right)(y x)$. If $\left(f_{S}+g_{S}\right)(x) \neq \varnothing$, then 
P. Murugadas and M.R.Thirumagal

$\left(f_{S}+g_{S}\right)(x)=\bigcup_{x=a+b}\left\{f_{S}(a) \cap g_{S}(b)\right\} \subseteq\left\{\bigcup_{x=a+b}\left\{f_{S}(y a) \cap g_{S}(y b)\right\}\right.$
$\subseteq \bigcup_{y x=c+d}\left\{f_{S}(c) \cap g_{S}(d)\right\}=\left(f_{S}+g_{S}\right)(y x)$.

Hence $f_{S}+g_{S}$ is a soft fuzzy left ideal of S.

Theorem 5.8. If $f_{S}, g_{S}$ are SI-left (right) ideals of a semiring $\mathrm{S}$, then $f_{S}{ }^{\circ} g_{S}$ is a SI-left (right) ideal of S.

Proof: Suppose $f_{S}, g_{S}$ are SI-left ideals of a semiring $S$ and $x, y \in S$. If $\left(f_{S}^{\circ} g_{S}\right)(x)=\varnothing$ or $\left(f_{S}^{\circ} g_{S}\right)(y)=\varnothing$,

then $\left(f_{S}^{\circ} g_{S}(x)\right) \cap\left(f_{S}^{\circ} g_{S}\right)(y)=\varnothing \subseteq\left(f_{S}^{\circ} g_{S}\right)(x+y)$.

If $\left(f_{S}^{\circ} g_{S}\right)(x) \neq \varnothing$ and $\left(f_{S}^{\circ} g_{S}(y)\right) \neq \varnothing$, then

$\left(f_{S}^{\circ} g_{S}\right)(x)=\bigcup_{x=a b}\left\{\left(f_{S}(a) \cap g_{S}(b)\right)\right\}$

$\left(f_{S}^{\circ} g_{S}\right)(y)=\bigcup_{y=c d}\left\{\left(f_{S}(c) \cap g_{S}(d)\right)\right\}$

$\left(f_{S}^{\circ} g_{S}\right)(x) \cap\left(f_{S}^{\circ} g_{S}\right)(y)=\left[\bigcup_{x=a b}\left\{\left(\left(f_{S}(a) \cap g_{S}(b)\right)\right\}\right] \cap\left[\bigcup_{y=c d}\left\{\left(\left(f_{S}(c) \cap g_{S}(d)\right)\right\}\right]\right.\right.$

$=\bigcup_{x=a b} \bigcup_{y=c d}\left[\left(\left(f_{S}(a) \cap g_{S}(b)\right)\right] \cap\left[\left[\left(\left(f_{S}(a) \cap g_{S}(b)\right] \subseteq \bigcup_{x+y=e f}\left[\left(\left(f_{S}(e) \cap g_{S}(f)\right)\right]\right.\right.\right.\right.\right.$

$=\left(f_{S}^{\circ} g_{S}\right)(x+y)$.

Again, if $\left(f_{S}^{\circ} g_{S}\right)(x)=\varnothing$ then $\left(f_{S}^{\circ} g_{S}\right)(x) \subseteq\left(f_{S}^{\circ} g_{S}\right)(y x)$.

If $\left(f_{S}^{\circ} g_{S}\right) \neq \varnothing$, then

$\left(f_{S}^{\circ} g_{S}\right)(x)=\bigcup_{x=a b}\left\{\left(f_{S}(a) \cap g_{S}(b)\right\} \subseteq \bigcup_{x=a b}\left\{\left(f_{S}(y a) \cap g_{S}(b)\right\}\right.\right.$

$\subseteq \bigcup_{y x=c d}\left\{\left(f_{S}(c) \cap g_{S}(d)\right\}=\left(f_{S}^{\circ} g_{S}\right)(y x)\right.$.

Hence $f_{S}{ }^{\circ} g_{S}$ is a SI- left ideal of S.

Theorem 5.9. Let $f_{S}$ be a soft set over $\mathrm{U}$. Then, if $f_{S}$ is a SI-left (right, two sided) ideal of $S$ over $\mathrm{U}, f_{S}$ is a SI-semiring over $\mathrm{U}$.

Proof: We give the proof for SI-ideals. Let $f_{S}$ be a SI-left ideal of $S$ over $U$. Then, $f_{S}(x+y) \supseteq f_{S}(x) \cap f_{S}(y)$ and $f_{S}(x y) \supseteq f_{S}(y)$ for all $x, y \in S$. Thus $f_{S}(x y) \supseteq$ $f_{S}(y) \supseteq f_{S}(x) \cap f_{S}(y)$, so $f_{S}$ is a SI-semiring over $\mathrm{U}$.

Theorem 5.10. Let $f_{S}$ be a SI-right ideal and $g_{S}$ a soft intersection left ideal of a semiring S. Then $f_{S} \circ g_{S} \subseteq f_{S} \cap g_{S}$. 


\section{Soft Intersection Ideals of Semiring}

Proof: Let $f_{S}$ and $g_{S}$ be a SI-right ideal of S. Then, since $f_{S}, g_{S} \subseteq \tilde{S}$ always holds, we have $f_{S} \circ g_{S} \tilde{\subseteq} f_{S} \circ \tilde{S} \subseteq f_{S}$ and $f_{S} \circ g_{S} \tilde{\subseteq} \tilde{S} \circ g_{S} \subseteq g_{S}$. Hence $f_{S} \circ g_{S} \tilde{\subseteq} f_{S} \cap g_{S}$.

Proposition 5.11. Let $f_{S}$ be a soft set over $U$ and $\alpha$ be a subset of $U$ such that $\alpha \in \operatorname{Im}\left(f_{S}\right)$. If $f_{S}$ is an SI-left (right) ideal of $S$ over $\mathrm{U}$, the $U\left(f_{S} ; \alpha\right)$ is a left (right) ideal of $S$ over $\mathrm{U}$.

\section{Soft intersection quasi-ideals of semiring}

Definition 6.1. A SI-semiring $f_{S}$ over $U$ is called a soft intersection quasi-ideal of $S$ over U.

(i) $f_{S}(x+y) \supseteq f_{S}(x) \cap f_{S}(y)$ for all $x, y \in S$

(ii) $\left(\tilde{S} \circ f_{S}\right) \cap\left(f_{S} \circ \tilde{S}\right) \subseteq f_{S}$.

Theorem 6.2. A soft set $f_{S}$ of a semiring $S$ is a soft intersection quasi-ideal of $S$ if and only if each nonempty level subset $U\left(f_{S} ; \alpha\right)$ of $f_{S}$ is a quasi-ideal of $\mathrm{S}$.

Proof: Suppose $f_{S}$ is a fuzzy quasi-ideal of S. Let $a, b \in U\left(f_{S} ; \alpha\right)$. Then $f_{S}(a) \supseteq \alpha$ and $f_{S}(b) \supseteq \alpha$. As $f_{S}(a+b) \supseteq f_{S}(a) \cap f_{S}(b)$, so $f_{S}(a+b) \supseteq \alpha$. Hence $a+b \in$ $U\left(f_{S} ; \alpha\right)$. Let $x \in U\left(f_{S} ; \alpha\right) \tilde{S} \cap \tilde{S} U\left(f_{S} ; \alpha\right)$. Then $x=\sum_{i=1}^{m} u_{i} r_{i}$ and $x=\sum_{k=1}^{p} s_{k} v_{k}$ for some $u_{i}, v_{k} \in U\left(f_{S} ; \alpha\right)$ and $r_{i}, s_{k} \in S$.

Now,

$$
\begin{aligned}
& f_{S}(x) \supseteq\left[\left(\tilde{S} \circ f_{S}\right) \cap\left(f_{S} \circ \tilde{S}\right)\right](x)=\left(\tilde{S} \circ f_{S}\right)(x) \cap\left(f_{S} \circ \tilde{S}\right)(x) \\
& =\bigcup_{x=\sum_{k=1}^{p} s_{k} v_{k}}\left[\tilde{S}\left(s_{k}\right) \cap f_{S}\left(v_{k}\right)\right] \cap \bigcup_{x=\sum_{i=1}^{m} u_{i} r_{i}}\left[f_{S}\left(u_{i}\right) \cap \tilde{S}\left(r_{i}\right)\right] \supseteq \alpha \cap \alpha=\alpha .
\end{aligned}
$$

.So, $f_{S}(x) \supseteq \alpha$. Thus, $x \in U\left(f_{S} ; \alpha\right)$. Hence $U\left(f_{S} ; \alpha\right) S \cap S U\left(f_{S} ; \alpha\right) \subseteq U\left(f_{S} ; \alpha\right)$.

Conversely, assume that each nonempty subset $U\left(f_{S} ; \alpha\right)$ of $S$ is a quasi-ideal of S. Let $a, b \in S$ be such that $f_{S}(a+b) \subset f_{S}(a) \cap f_{S}(b)$. Take $\alpha \subseteq U$ such that $f_{S}(a+b) \subset \alpha \subseteq f_{S}(a) \cap f_{S}(b)$. Then $a, b \in U\left(f_{S} ; \alpha\right)$ but $a+b \notin U\left(f_{S} ; \alpha\right)$, a contradiction. Hence $f_{S}(a+b) \supseteq f_{S}(a) \cap f_{S}(b)$.

Let $x \in S$. If possible let $f_{S}(x) \subset\left[\left(f_{S} \circ S\right) \cap\left(S \circ f_{S}\right)\right](x)$. Take $\alpha \subseteq U$ such that $f_{S}(x) \subset \alpha \subseteq\left[\left(f_{S} \circ \tilde{S}\right) \cap\left(\tilde{S} \circ f_{S}\right)\right](x)$. If $\left[\left(f_{S} \circ \tilde{S}\right) \cap\left(\tilde{S} \circ f_{S}\right)\right](x) \supseteq \alpha$, then 
P. Murugadas and M.R.Thirumagal

$$
\left[\left(\tilde{S} \circ f_{S}\right) \cap\left(f_{S} \circ \tilde{S}\right)\right](x)=\bigcup_{x=\sum_{k=1}^{p} s_{k} v_{k}}\left[\tilde{S}\left(s_{k}\right) \cap f_{S}\left(v_{k}\right)\right] \cap \bigcup_{x=\sum_{i=1}^{m} u_{i} r_{i}}\left[f_{S}\left(u_{i}\right) \cap \tilde{S}\left(r_{i}\right)\right] .
$$

Hence, $\bigcup_{x=\sum_{k=1}^{p} s_{k} v_{k}}\left[\tilde{S}\left(s_{k}\right) \cap f_{s}\left(v_{k}\right)\right] \supseteq \alpha$ and $\bigcup_{x=\sum_{i=1}^{m} s_{k} v_{k}}\left[f_{S}\left(u_{i}\right) \cap \tilde{S}\left(r_{i}\right)\right] \supseteq \alpha$.

so, $f_{S}\left(u_{i}\right) \supseteq \alpha, f_{S}\left(v_{k}\right) \supseteq \alpha$, that is, $u_{i}, v_{k} \in U\left(f_{s} ; \alpha\right)$ for all $i, k$. Thus

$\sum_{i=1}^{m} u_{i} r_{i} \in U\left(f_{S} ; \alpha\right) S$ and $\sum_{k=1}^{p} s_{k} v_{k} \in S U\left(f_{S} ; \alpha\right)$. This implies $x \in U\left(f_{S} ; \alpha\right) S \cap S U$ $\left(f_{S} ; \alpha\right) \subseteq U\left(f_{S} ; \alpha,\right)$ and hence $x \in U\left(f_{S} ; \alpha\right)$, that is $f_{S}(x) \supseteq \alpha$, a contradiction.

Hence $\left(f_{S} \circ \tilde{S}\right) \cap\left(\tilde{S} \circ f_{S}\right) \tilde{\subseteq} f_{S}$. Thus $f_{S}$ is a soft intersection quasi-ideal of $\mathrm{S}$.

Corollary 6.3. Let $\mathrm{Q}$ be a nonempty subset of a semiring $\mathrm{S}$. Then $Q$ is a quasi-ideal of $S$ if and only if the characteristic function $\tilde{S}$ of $Q$ is a fuzzy quasi-ideal of $\mathrm{S}$.

Proposition 6.4. The intersection of any two SI-quasi-ideals of a semiring $S$ is a SIquasi-ideal of $\mathrm{S}$.

Proof: Let $f_{S}, g_{S}$ be SI-semiring quasi-ideals of a semiring $S$ and $x, y \in S$. Then

$\left(f_{S} \cap g_{S}\right)(x+y)=f_{S}(x+y) \cap g_{S}(x+y) \supseteq\left[f_{S}(x) \cap f_{S}(y)\right] \cap\left[g_{S}(x) \cap g_{S}(y)\right]$

$=\left[f_{S}(x) \cap g_{S}(x)\right] \cap\left[f_{S}(y) \cap g_{S}(y)\right]=\left(f_{S} \cap g_{S}\right)(x) \cap\left(f_{S} \cap g_{S}\right)(y)$.

Also,

$\left(\left(f_{S} \cap g_{S}\right) \circ \tilde{S}\right) \cap\left(\tilde{S} \circ\left(f_{S} \cap g_{S}\right)\right) \subseteq\left(f_{S} \circ \tilde{S}\right) \cap\left(\tilde{S} \circ f_{S}\right) \subseteq f_{S}$.

$\left.\left(\left(f_{S} \cap g_{S}\right) \circ \tilde{S}\right) \tilde{S} \circ\left(f_{S} \cap g_{S}\right)\right) \subseteq\left(g_{S} \circ \tilde{S}\right) \cap\left(\tilde{S} \circ g_{S}\right) \subseteq g_{S}$.

Thus $\left(\left(f_{S} \cap g_{S}\right) \circ \tilde{S}\right) \cap\left(\tilde{S} \circ\left(f_{S} \cap g_{S}\right)\right) \subseteq f_{S} \cap g_{S}$.

Corollary 6.5. Let $f_{S}$ and $g_{S}$ be SI- right and SI- left ideals of a semiring S, respectively. Then $f_{S} \cap g_{S}$ is a SI- quasi-ideal of $\mathrm{S}$.

\section{Soft intersection bi-ideals of semiring}

Definition 7.1. A soft set over $U$ is called a SI- bi-ideals of $S$ over $U$ if

(1) $f_{S}(x+y) \supseteq f_{S}(x) \cap f_{S}(y)$

(2) $\left.f_{S}(x y) \supseteq f_{S}(x) \cap f_{S}(y)\right)$

(3) $f_{S}(x y z) \supseteq f_{S}(x) \cap f_{S}(z)$ for all $x, y, z \in S$.

Example 7.2. Consider the semiring $S=\{0, a, b, c\}$ defined by the following table: 
Soft Intersection Ideals of Semiring

\begin{tabular}{|c|l|l|l|l|}
\hline$\oplus$ & 0 & $\mathrm{a}$ & $\mathrm{b}$ & $\mathrm{c}$ \\
\hline 0 & 0 & $\mathrm{a}$ & $\mathrm{b}$ & $\mathrm{c}$ \\
\hline $\mathrm{a}$ & $\mathrm{a}$ & $\mathrm{b}$ & $\mathrm{c}$ & $\mathrm{a}$ \\
\hline $\mathrm{b}$ & $\mathrm{b}$ & $\mathrm{c}$ & $\mathrm{a}$ & $\mathrm{b}$ \\
\hline $\mathrm{c}$ & $\mathrm{c}$ & $\mathrm{a}$ & $\mathrm{b}$ & $\mathrm{c}$ \\
\hline
\end{tabular}

\begin{tabular}{|l|l|l|l|l|}
\hline$\odot$ & 0 & $\mathrm{a}$ & $\mathrm{B}$ & $\mathrm{c}$ \\
\hline 0 & 0 & 0 & 0 & 0 \\
\hline $\mathrm{A}$ & 0 & $\mathrm{a}$ & $\mathrm{B}$ & $\mathrm{c}$ \\
\hline $\mathrm{B}$ & 0 & $\mathrm{~b}$ & $\mathrm{~B}$ & $\mathrm{c}$ \\
\hline $\mathrm{C}$ & 0 & $\mathrm{c}$ & $\mathrm{C}$ & $\mathrm{c}$ \\
\hline
\end{tabular}

Define the soft set $f_{S}$ over $U=Z_{4}$ such that $f_{S}(0)=\{\overline{0}, \overline{1}, \overline{2}\}, f_{s}(a)=\{\overline{0}, \overline{1}\}$, $f_{S}(b)=\{\overline{0}\}, f_{S}(c)=\{\overline{1}, \overline{2}\}$. Clearly $f_{S}$ is a SI-Bi-ideal of $S$ over U.

Theorem 7.3. Let $f_{S}$ be a soft set over $\mathrm{U}$. Then, $f_{S}$ is a SI-bi-ideal of $S$ over $U$ if and only if

(i) $f_{S}+f_{S} \tilde{\subseteq} f_{S}$

(ii) $f_{S} \circ f_{S} \tilde{\subseteq} f_{S}$

(iii) $f_{S} \circ \tilde{S} \circ f_{S} \subseteq f_{S}$.

Proof: Let $f_{S}$ be a SI- bi-ideal of $S$ and $x \in S$. Then

$$
\begin{aligned}
& (1)\left(f_{S}+f_{S}\right)(x)=\bigcup_{x=y+z}\left\{f_{S}(y) \cap f_{S}(z)\right\} \\
\subseteq & \bigcup_{x=y+z} f_{S}(y+z)=f_{S}(x) .
\end{aligned}
$$

Therefore $f_{S}+f_{S} \subseteq f_{S}$.

(2) $f_{S} \circ f_{S} \subseteq f_{S}$ is evident.

(3) Let $s \in S$. If $\left(f_{S} \circ \tilde{S} \circ f_{S}\right)(s)=\varnothing$, then $f_{S} \circ \tilde{S} \circ f_{S} \subseteq f_{S}$. Otherwise, there exist element $x, y, p, q \in S$ such that $s=x y$ and $x=p q$.

Then, since $f_{S}$ is an SI-bi-ideal of $S$ over $\mathrm{U}$, we have

$$
\begin{aligned}
& \left(f_{S} \circ \tilde{S} \circ f_{S}\right)(s)=\left[\left(f_{S} \circ \tilde{S} \circ f_{S}\right)(s)\right]=\bigcup_{s=p q y}\left(f_{S}(p) \cap f_{S}(y)\right) \subseteq \bigcup_{s=p q y} f_{S}(p q y) \\
& =f_{S}(x y)=f_{S}(s)
\end{aligned}
$$

Hence, $f_{S} \circ \tilde{S} \circ f_{S} \subseteq f_{S}$. Here, note that if $x \neq p q$, then $\left(f_{S} \circ \tilde{S}^{\circ} f_{S} \subseteq f_{S}\right)(a)=\varnothing$, and so $\left(f_{S} \circ \tilde{S} \circ f_{S}\right)=\varnothing \subseteq f_{S}(s)$.

Conversely, assume (1) and (2). By theorem 4.1, $f_{S}$ is a SI-semiring of S. Let $x, y, z \in S$ and $s=x y z$. Then, since $f_{S} \circ \tilde{S} \circ f_{S} \subseteq f_{S}$, we have $f_{S}(x y z)=f_{S}(s) \supseteq\left(f_{S} \circ \tilde{S} \circ f_{S}\right)(s)=\left[\left(f_{S} \circ \tilde{S}\right) \circ f_{S}\right](s)$ 
P. Murugadas and M.R.Thirumagal

$$
\begin{aligned}
& =\bigcup_{s=(x y) z}\left[\left(\left(f_{S} \circ \tilde{S}(x y) \cap f_{S}(z)\right)\right] \supseteq\left(f_{S} \circ \tilde{S}\right)(x y) \cap f_{S}(z)=\left[\bigcup\left(f_{S}(x) \cap \tilde{S}(y)\right)\right] \cap f_{S}(z)\right. \\
& \supseteq\left(\left(f_{S}(x) \cap \tilde{S}\right)(y)\right) \cap f_{S}(z)=\left(f_{S}(x) \cap U\right) \cap f_{S}(z)=f_{S}(x) \cap f_{S}(z) .
\end{aligned}
$$

Thus, $f_{S}$ is a SI-bi-ideal of $S$ over $\mathrm{U}$.

Theorem 7.4. Let $X$ be a nonempty subset of a semiring S. Then, $X$ is a bi-ideal of $S$ if and only if $S_{X}$ is a SI-bi-ideal of $S$ over $\mathrm{U}$.

Proof: Assume that $X$ is a bi-ideal of $\$ S, \$$ that is, $X+X \subseteq X, X X \subseteq X$ and $X S X \subseteq X$. Then, we have

$S_{X}+S_{X}=S_{X+X} \subseteq S_{X}$ and

$S_{X} \circ S_{X}=S_{X X} \subseteq S_{X}$ (since $\mathrm{x} \subseteq \subseteq X$ ). Thus, $S_{X}$ is a SI-semiring over $\mathrm{U}$.

Moreover $S_{X} \circ \tilde{S} \circ S_{X}=S_{X} \circ S_{s} \circ S_{X}=S_{X S X} \subseteq S_{X}($ since $X S X \subseteq X)$

This means that $S_{X}$ is a bi-ideal of $\mathrm{S}$.

Conversely, let $S_{X}$ be an SI-bi-ideal of S. It means that $S_{X}$ is a SI-semiring. Let $x \in X+X$. Then, $S_{X}(x) \supseteq\left(S_{X}+S_{X}\right)(x)=S_{X+X}(x)=U \Rightarrow x \in X \Rightarrow X+X \subseteq X$ and let $x \in X X$. Then, $S_{X}(x) \supseteq\left(S_{X}{ }^{\circ} S_{X}\right)(x)=S_{X X}(x)=U \Rightarrow x \in X \Rightarrow X X \subseteq X$.

Therefore $X$ is a subsemiring of $S$. Next, let $y \in X S X$. Thus

$S_{X}(y) \supseteq\left(S_{X} \circ S \circ S_{X}\right)(y)=\left(S_{X} \circ S_{s} \circ S_{X}\right)(y)=S_{X S X}(y)=U$ and so $y \in X$. Thus $X S X \subseteq X$ and $X$ is a bi-ideal of $\mathrm{S}$.

Theorem 7.5. Every SI-left (right, two sided) ideal of a semiring $S$ over $U$ is a SI-biideal of $S$ over $\mathrm{U}$.

Proof: Let $f_{S}$ be a SI-left (right, two sided) ideal of a semiring $S$ over $U$ and $x, y, z \in S$. Then $f_{S}$ is as SI-semiring by Theorem (5.6.). Moreover,

$f_{S}(x y z)=f_{S}((x y) z) \supseteq f_{S}(z) \supseteq f_{S}(x) \cap f_{S}(z)$. Thus $f_{S}$ is a SI-bi-ideal of S.

Theorem 7.6. Let $f_{S}$ be any SI-ideal of a semiring $S$ and $g_{S}$ any SI-bi-ideal of S. $f_{S} \circ g_{S}$ and $g_{S} \circ f_{S}$ are SI-bi-ideals of S.

Proof: To show that $f_{S} \circ g_{S}$ is a SI-bi-ideal of S, first we need to show that $f_{S} \circ g_{S}$ is a SI-semiring. Thus

$$
\begin{aligned}
& \left(f_{S} \circ g_{S}\right) \circ\left(f_{S} \circ g_{S}\right)=f_{S} \circ\left(g_{S} \circ\left(f_{S} \circ g_{S}\right)\right) \\
& \tilde{\subseteq} f_{S} \circ\left(g_{S} \circ\left(\circ g_{S}\right)\right)\left(\text { since } f_{S} \tilde{\subseteq} \tilde{S}\right)=f_{S} \circ\left(g_{S} \circ\left(\tilde{S} \circ g_{S}\right)\right) \\
& \tilde{\subseteq} f_{S} \circ g_{S}\left(\text { since } g_{S} \circ\left(\tilde{S} \circ g_{S}\right) \tilde{\subseteq} g_{S},\left(g_{S} \text { being SI-bi-ideal }\right)\right.
\end{aligned}
$$

Hence by Theorem 4.1, $f_{S} \circ g_{S}$ is a SI-semiring over U. Moreover we have 
Soft Intersection Ideals of Semiring

$\left(f_{S} \circ g_{S}\right) \circ \tilde{S} \circ\left(f_{S} \circ g_{S}\right)=f_{S} \circ\left(g_{S} \circ\left(\tilde{S} \circ f_{S}\right) \circ g_{S}\right)$

$\tilde{\subseteq} f_{S} \circ\left(g_{S} \circ\left(\tilde{S} \circ g_{S}\right)\right)\left(\right.$ since $\left.S \circ f_{S} \tilde{\subseteq} \tilde{S}\right) \tilde{C} f_{S} \circ g_{S}$.

Thus, it follow that $f_{S} \circ g_{S}$ is a SI-bi-ideal of S. It can be proved in a similar way that $g_{S}{ }^{\circ} f_{S}$ is a SI-bi-ideal of $S$ over U.

Theorem 7.7. Intersection of a non-empty collection of SI- bi-ideal of S over $U$ is also a SI- bi-ideal of $\mathrm{S}$ over $\mathrm{U}$.

Proof: The proof follows by routine verifications.

Theorem 7.8. Let $\left\{f_{i}: i \in I\right\}$ be a family of SI- bi-ideal of $S$ over $U$ such that $f_{i} \subseteq f_{j}$ or $f_{j} \subseteq f_{i}$ for $i, j \in I$. Then $\bigcup_{i \in I} f_{i}$ is a SI- bi-ideal of $S$ over $\mathrm{U}$.

Proof: The proof follows by routine verifications

Theorem 7.9. In a semiring every SI- quasi ideals are SI- bi-ideals.

\section{Conclusion}

Through this paper, SI- semiring, SI- left (right, two-sided) ideals of semiring, SI- quasi ideal of semiring and SI- bi- ideal of semirings are studied and properties pertaining to them elicited.

Acknowledgement. The first author is thankful to the University Grants Commission, New Delhi-110021, India, for providing OBC National fellowship under grant no:F.41/2016-17/NFO-2015-17-OBC-TAM-29759/(SA-III/Website) 01.04.2016

\section{REFERENCES}

1. N.C.Agman and S.Enginoglu, Soft set theory and uni-int decision making, Eur. J. Oper. Res., 207 (2010) 848 - 855.

2. N.C.Agman and S.Enginoglu, Soft matrix theory and its decision making, Comput. Math. Appl., 59 (2010) 3308 - 3314.

3. J.Ahsan, K.Saifullah and M.Farid Khan, Fuzzy semirings, Fuzzy Sets and Systems, 60 (1993) 309-320.

4. M.I.Ali, F.Feng, X.Liu, W.K.Min and M.Shabir, On some new operations in soft set theory, Comput. Math. Appl., 57 (2009) 1547-1553.

5. F.Fenga, Y.B.Jun and X.Zhao, Soft semirings, Comput. and Math. Appl., 56 (2008) 2621-628.

6. F.Feng, X.Y.Liu, V.L.-Fotea and Y.B.Jun, Soft sets and soft rough sets, Inform. Sci., 181 (2011) 1125-1137.

7. K.Glazek, A Guide to the Literature on Semirings and their Applications in Mathematics and Information Sciences, Kluwer, Dordrecht, 2002.

8. J.S.Golan, Semirings and Affine Equations over Them: Theory and Applications, Kluwer, Dordrecht, 2003.

9. M.Henriksen, Ideals in semirings with commutative addition, Amer. Math. Soc. Notices, 6 (1958) 321.

10. K.Iizuka, On the Jacobson radical of a semiring, Tohoku Math. J., 11 (2) (1959) 409 421. 


\section{P. Murugadas and M.R.Thirumagal}

11. P.K.Maji, A.R.Roy and R.Biswas, An application of soft sets in a decision making problem, Comput. Math. Appl., 44 (2002)1077-1083.

12. P.K.Maji, R.Biswas and A.R.Roy, Soft set theory, Comput. Math. Appl., 45 (2003) 555-562.

13. D.A.Molodtsov, Soft set theory-first results, Comput. Math. Appl., 37 (1999) 19-31.

14. A.Rosenfeld, Fuzzy groups, J. Math. Anal. Appl., 35 (1971) 512 - 517.

15. A.Sezgin and A.O.Atag"un, On operations of soft sets, Comput. Math. Appl., 61 (2011) 1457-1467.

16. L.A.Zadeh, Fuzzy sets, Information and Control, 8 (1995) 338-353.

17. Y.Zou and Z.Xiao, Data analysis approaches of soft sets under incomplete information, Knowl. Base. Syst., 21 (2008) 941-945. 